\title{
Severe child obesity levels at record high in 10- to 11-year-olds
}

The level of severe obesity in children aged 10 to 11 years in England has reached the highest point since records began, according to new figures published on 24 July 2018 by Public Health England (PHE). ${ }^{1}$

PHE's analysis of the National Child Measurement Programme (NCMP) between 2006 to 2007 and 2016 to 2017 shows trends in severe obesity for the first time, an issue which is often linked to accompanying poor dental health.

The NCMP captures the height and weight of more than one million children in Reception (aged 4 to 5 years) and Year 6 (aged 10 to 11 years) in school each year.

Analysis showed that one in 25 children aged 10 and 11 were severely obese, equating to around 22,000 children.

Although overall childhood obesity levels have remained steady in recent years, the new figures showed levels of severely obese children have been rising over the past decade.

In addition, the findings showed health inequalities continued to widen with the prevalence of excess weight, obesity, overweight and severe obesity being higher in the most deprived areas compared to the least deprived.

The analysis also showed:

- An upward trend of excess weight, obesity and severe obesity in Year 6 children

- A downward trend of excess weight, overweight, obesity and severe obesity in Reception age boys

- A downward trend of underweight in Reception age boys and girls, and Year 6 girls.

Dr Alison Tedstone, chief nutritionist at PHE, said: 'The rise in severe obesity and widening health inequalities highlight why bold measures are needed to tackle this threat to our children's health.

These trends are extremely worrying and have been decades in the making - reversing them will not happen overnight.'

As part of its work to reduce childhood obesity, PHE is working with the food industry to cut $20 \%$ of sugar from everyday products by 2020 , and $20 \%$ of calories by 2024 .

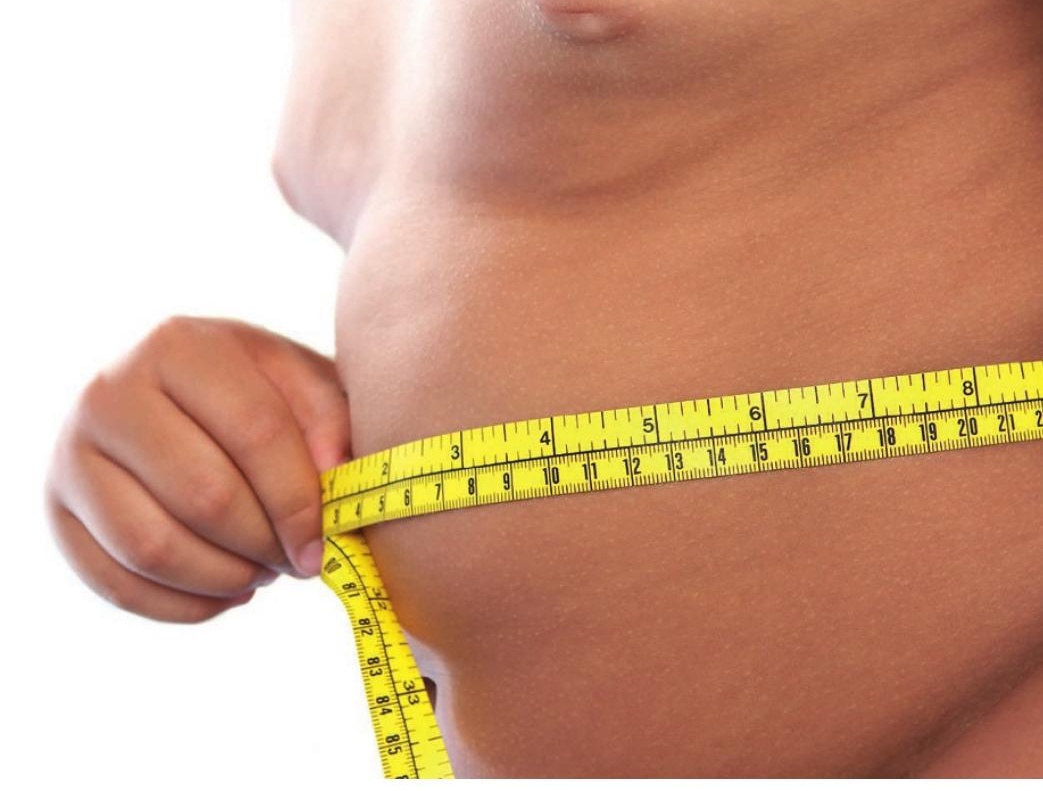

The Obesity Health Alliance (OHA), a coalition of more than 40 charities, medical royal colleges and campaign groups including the BDA working together to share expertise and support the Government in tackling overweight and obesity in the UK, said the figures were a cause for concern.

Caroline Cerny, Alliance Lead for the OHA, said: 'It's very concerning that the number of children with a weight that is classified as severely obese is now at an all-time high. Children with obesity are five times more likely to have obesity as adults, putting them at risk of diseases including Type 2 diabetes, cancer, heart and liver disease, as well as associated mental health conditions.

'The Government has recently set out some bold plans with an ambition of halving childhood obesity by 2030, including a 9 pm watershed on junk food adverts. We need all these measures to be fully and swiftly implemented to help ensure all children have the healthiest possible start in life.'

Public Health England. Trends in children's body mass index between 2006/07 and 2016/17: Analysis of data from the National Child Measurement Programme (July 2018). https://app.box.com/s/og3q86aqejc99okxe9xyvpfvo21xai21/ file/306723044116 (accessed on 24 July 2018).

\section{BDJ RESEARCH INSIGHTS}

- Research insights from across the BDJ Portfolio

- Providing busy readers with valuable context including article summaries and expert commentary

Catch up here: http://bit.ly/BDJResearchlnsights

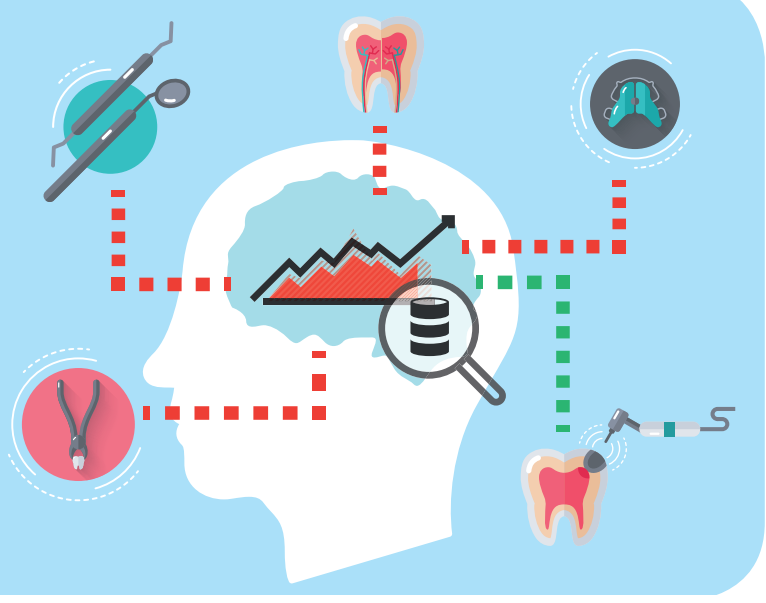

